\title{
C COBENGE \\ ESTUDO PARA VALIDAÇÃO DO PERFIL DO EGRESSO DO CURSO DE ENEGNHARIA CIVIL DA UNIFEI
}

DOI: 10.37702/2175-957X.COBENGE.2021.3511

Adinele Gomes Guimarães - adinele@unifei.edu.br

Universidade Federal de Itajubá

Av BPS 1303

37500-903 - Itajubá - MG

André Luiz Vivan - andrevivan@unifei.edu.br

Universidade Federal de Itajubá

Rua Heitor Pires 60

37500-000 - Itajubá - MG

Daniele Ornaghi Sant Anna - ornaghi@unifei.edu.br

Universidade Federal de Itajubá

Alberto José Pereira 211

37502-332 - Itajubá - MG

FERNANDO DAS GRAÇAS BRAGA DA SILVA - ffbraga.silva@gmail.com Universidade Federal de Itajubá

AV BPS 1303

37500-903 - ITAJUBÁ - MG

Josiane Palma Lima - jplima@unifei.edu.br

Universidade Federal de Itajubá

Praça Roberto Carneiro 45

37500-365 - Itajubá - MG

Marcia Viana Lisboa Martins - marciaviana@unifei.edu.br

UNIFEI

Rua Dr. Silvestre Ferraz, 880880

37500-054 - Itajubá - MG

Maria Rachel de Araújo Russo - rachelrusso99@unifei.edu.br

Universidade Federal de Itajubá

Rua João Puliti 650

37500-095 - Itajubá - MG 
Nívea Adriana Dias Pons - npons@unifei.edu.br

Universidade Federal de Itajubá

Rua Antônio Correa Cardoso 101

37501-064 - Itajubá - MG

Paulo Cesar Gonçalves - paulocg9@unifei.edu.br

Universidade Federal de Itajubá

Rua Antônio Dastre 167

37501-057 - Itajubá - MG

Valquíria claret dos Santos - valquiria@unifei.edu.br

Universidade Federal de Itajubá

Coronel Joaquim Francisco 78

37501-052 - Itajubá - MG

Resumo: $O$ curso de Engenharia Civil da Universidade Federal de Itajubá (Unifei) foi proposto no programa REUNI e teve seu início em 2011. Em 2019, motivados pelas discussões e alteração nas Diretrizes Curriculares Nacionais (DCNs) dos cursos de graduação em Engenharia, o Núcleo Docente Estruturante da Engenharia Civil (NDE-ECI), iniciou o processo de reestruturação do Projeto Pedagógico do Curso (PPC). O ponto de partida foi as discussões sobre o perfil do egresso, culminando na definição das competências que pretendem ser desenvolvidas aos alunos durante sua formação na universidade. O NDE-ECI propôs que o egresso deveria ser habilitado a atuar nos cinco campos do conhecimento da Engenharia Civil, sendo eles: Construção Civil; Estruturas; Geotecnia, Hidrotecnia e Transportes. Assim ficaram estabelecidas 14 competências, sendo as oito primeiras as competências gerais das DCNs, estabelecidas no Art $4^{\circ}$ da Resolução CNE/CES N 2 de 24 de abril de 2019 e as outras seis definidas pelo NDE-ECl, sendo uma para cada das áreas de atuação profissional e uma para a concepção original do curso, de formação de profissional com foco em tecnologias modernas e preocupado com as questões ambientais. Com o objetivo de validar o perfil do egresso proposto pelo NDE, foi aplicado um questionário com os egressos do curso. No questionário os egressos da Engenharia Civil opinaram, em uma escala de 1 (pior) a 5 (melhor), sobre as grandes áreas de conhecimentos básicos e específicos, avaliando a formação que receberam durante o curso e a importância delas na atuação que desempenham profissionalmente. Os resultados demostraram que a maioria das áreas está satisfatória em termos da capacitação e é importante para o mercado de trabalho. O levantamento realizado corrobora com as competências específicas propostas 
pelo NDE-ECl, visto que as áreas de maior pontuação estão relacionadas com os campos indicados nelas. Conclui-se que o perfil do egresso do curso de graduação em Engenharia Civil da Unifei foi definido com base nos campos de exercício profissional, na atuação dos egressos, nas demandas de mercado e nas discussões com os professores.

Palavras-chave: egresso, projeto pedagógico, construção civil, estruturas, geotecnia, hidrotecnia, transportes, sustentabilidade 


\section{ESTUDO PARA VALIDAÇÃO DO PERFIL DO EGRESSO DO CURSO DE ENEGNHARIA CIVIL DA UNIFEI}

\section{INTRODUÇÃO}

A Universidade Federal de Itajubá (Unifei) foi criada em 2002, pela Lei $n^{\circ} 10.435$, a partir da transformação da Escola Federal de Engenharia de Itajubá (Efei). A instituição foi fundada em 1913 por iniciativa pessoal de Theodomiro Carneiro Santiago, que almejava, sobretudo, formar engenheiros práticos, capacitados para serem úteis à indústria nacional, à sociedade e à grandeza do país.

Ao longo dos anos a instituição empreendeu-se a políticas de expansão para poder oferecer um atendimento mais amplo e diversificado à demanda nacional e, sobretudo, regional, de formação de profissionais na área tecnológica. Em 1998 os cursos de graduação em Engenharia Ambiental e de Engenharia Hídrica, ambos abrigados no Instituto de Recursos Naturais (IRN) da Unifei, foram autorizados. Deste modo o cenário ficou favorável para a implementação do curso de Engenharia Civil, considerando a forte relação entre estes cursos, e que grande parte de suas infraestruturas é comum e poderia ser aproveitada.

O curso de Engenharia Civil da Unifei foi então proposto no programa Reestruturação e Unificação das Universidades (REUNI), considerando o aproveitamento de recursos humanos e estruturas físicas existentes na instituição. O curso apresentou-se como uma peça relevante não só para fortalecimento da tradição da universidade, considerando seu arcabouço na engenharia, como também complementou uma lacuna desta área tão necessária para o desenvolvimento regional e nacional.

O primeiro processo seletivo do curso foi realizado em 2011. E em 27 de dezembro de 2018 teve seu reconhecimento pelo Ministério da Educação, por meio da Portaria № 920. Passados então dez anos da criação do curso de Engenharia Civil na Unifei, e tendo sido contratados docentes para as áreas específicas do curso, pode-se repensar e almejar a atualização do seu projeto pedagógico, bem como adequar o perfil do egresso, cuja dinâmica de definição é apresentada neste artigo.

\section{O PROJETO PEDAGÓGICO DO CURSO}

O primeiro Projeto Pedagógico de Curso (PPC) de graduação em Engenharia Civil da Unifei baseava-se na proposta de um curso inovador voltado para a sustentabilidade, no qual o aluno e o professor estivessem intimamente engajados: a) na responsabilidade social e ambiental, através do conhecimento e conscientização do estudante acerca dos impactos positivos e negativos do desenvolvimento econômico, de forma a pautar suas futuras ações como engenheiro civil na sustentabilidade ambiental; b) na busca do conhecimento através da pesquisa, do questionamento crítico, do trabalho em grupo e da utilização de ferramentas como laboratórios, instrumentação, consultas à biblioteca e aos meios eletrônicos disponíveis; c) na construção de novas propostas, projetos desafiadores e avaliação crítica de resultados; d) no compartilhamento de conhecimento e atividades com empresas, de modo a enriquecer o espaço universitário com recursos humanos e instrumentais; e e) com disciplinas voltadas para a sustentabilidade.

Em 2019, motivado pelas discussões e proposições de alteração nas Diretrizes Curriculares Nacionais (DCNs) dos cursos de graduação em Engenharia, o Núcleo Docente Estruturante da Engenharia Civil da Unifei (NDE-ECI), iniciou o processo de 
reestruturação de seu projeto pedagógico. O ponto de partida deste processo foram as discussões sobre o perfil do egresso, culminando na definição das competências que pretendem ser desenvolvidas aos alunos durante sua formação na universidade, sejam de caráter técnico ou pessoal. Este projeto encontra-se em elaboração, portanto este artigo restringe-se ao relato da primeira fase do novo PPC.

\section{PERFIL DO EGRESSO}

\section{1 Áreas de atuação}

Após diversos encontros do NDE-ECl, definiu-se que o engenheiro civil graduado pela Unifei deveria ser um profissional apto a atuar em todo o ciclo de vida e contexto do projeto de produtos e de seus componentes, sistemas e processos produtivos, inclusive inovando-os; e também atuar no ciclo de vida e contexto de empreendimentos, inclusive em gestão e manutenção, conforme itens I e II do Art. $5^{\circ}$ da Resolução CNE/CES $N^{\circ} 2$ (MINISTÉRIO DA EDÚCAÇÃO, 2019), que institui as DCNs do curso de graduação em engenharia.

Ainda em conformidade com o artigo supracitado, o NDE-ECl propôs que a atuação do egresso deve acontecer ser nos cinco campos do conhecimento da Engenharia Civil e seus correlatos, tais como: Construção Civil; Estruturas; Geotecnia, Hidrotecnia e Transportes. Foram então estabelecidas cinco competências, sendo uma para cada área de atuação profissional, a saber:

- Construção Civil: projetar, desenvolver, organizar e gerenciar o ciclo de vida da obra, visando sua adequação e otimização econômica, técnica, legal e ambiental, por meio dos conceitos e técnicas do planejamento e gestão da obra e sua melhoria contínua, da tecnologia, comportamento, execução e aplicação dos materiais de construção e dos sistemas construtivos.

- Estruturas: analisar, dimensionar e projetar estruturas a partir de métodos de dimensionamentos clássicos, atendendo às prescrições das normas brasileiras pertinentes. Detalhar e supervisionar as soluções dos principais elementos em estruturas usuais. Compreender e aplicar as prescrições das normas brasileiras relacionadas ao projeto e execução da estrutura. Identificar e compreender os fundamentos dos materiais das estruturas usuais no que se refere ao comportamento estrutural.

- Geotecnia: avaliar, conceber e executar sistemas, métodos e projetos no âmbito da engenharia geotécnica. Desempenhar atividades técnicas, científicas e profissionais relacionadas às áreas de investigação de subsolo, fundações, barragens, obras de terra, estruturas de contenção, obras subterrâneas e estabilidade de taludes e encostas, entre outras que fazem uso do conhecimento da mecânica dos solos e geologia.

- Hidrotecnia (Hidráulica, Hidrologia e Saneamento): conceber, projetar, planejar e gerenciar projetos de recursos hídricos e saneamento, compreendidos pelo aproveitamento e utilização de recursos naturais, tais como hidráulica de barragens, canais, reservatórios, sistemas de irrigação e drenagem, e obras portuárias e costeiras, sistema de abastecimento de água, sistema de esgotamento sanitário, limpeza urbana e manejo dos resíduos sólidos, drenagem e manejo das águas pluviais e sistemas hidráulicos e sanitários prediais.

- Transportes: projetar, construir e manter a infraestrutura viária e de transportes; estudar, planejar, operar e controlar o tráfego de veículos e a integração dos vários modos de transportes; desenvolver planos e projetos com ênfase na capacidade e racionalização dos sistemas de transportes e da mobilidade urbana sustentável. 
Além destes, para manter a concepção original do curso, na busca de um engenheiro com foco em tecnologias modernas e preocupado com as questões ambientais, também foi incluída a seguinte competência:

- Desenvolver projetos seguros e econômicos com foco na sustentabilidade, eficiência hídrica e energética e uso racional dos recursos naturais.

Dessa forma, ao longo de sua formação, o aluno da Engenharia Civil da Unifei será auxiliado no desenvolvimento de 14 competências, sendo as oito primeiras as competências gerais das DCNs, estabelecidas no Art $4^{\circ}$ da Resolução CNE/CSE $N^{\circ} 2$ (MINISTÉRIO DA EDUCAÇÃO, 2019), e as outras seis definidas pelo NDE-ECI, permitindo que o seu egresso possa atuar como profissional liberal ou em empresas públicas ou privadas e desenvolver pesquisas científicas e tecnológicas nos diferentes segmentos da Engenharia Civil.

\subsection{Questionário com egressos}

Com o objetivo de validar o perfil do egresso proposto pelo NDE, foi aplicado um questionário aos egressos do curso. Para essa consulta foi utilizado um formulário on-line através do Google Forms, por se tratar de uma ferramenta gratuita, de fácil acesso e que permite o arquivamento das respostas com segurança, sendo atualmente um método muito empregado na coleta de informações de diferentes trabalhos científicos. A divulgação do questionário ocorreu pelo correio eletrônico e pelas redes sociais.

Foram obtidas 42 respostas dos 135 concluintes da graduação em Engenharia Civil na Unifei até o primeiro semestre de 2020 , ou seja, aproximadamente $31 \%$ dos egressos participaram da pesquisa, quantitativo que foi considerado satisfatório. Quanto ao ano de conclusão do curso, houve uma maior participação dos egressos dos últimos dois anos, conforme Figura 1.

Figura 1 - Resultado quanto ao ano de conclusão do curso

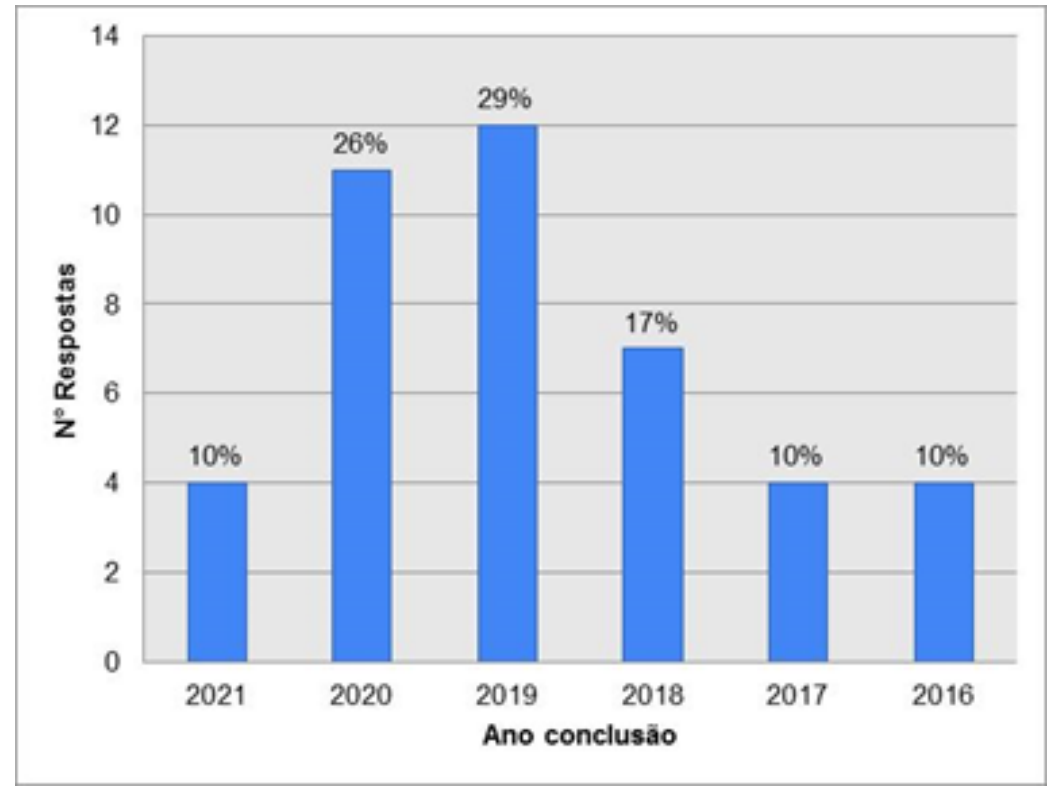

$\mathrm{Na}$ Figura 2 encontra-se o resultado geral quanto ao emprego atual dos respondentes. Observa-se que a maioria dos egressos encontra-se atuando em setores da engenharia e a menor porcentagem foi para programas de pós-graduação.

No questionário os egressos da Engenharia Civil opinaram, em uma escala de 1 (pior) a 5 (melhor), sobre as grandes áreas de conhecimentos básicos e específicos, avaliando a formação que receberam durante o curso e a importância delas na atuação 
que desempenham profissionalmente. Para análise das respostas foi elaborado um gráfico das médias das notas, correlacionando atuação e formação, como mostrado na Figura 3.

Figura 2 - Resultado dos egressos quanto a atuação profissional atual

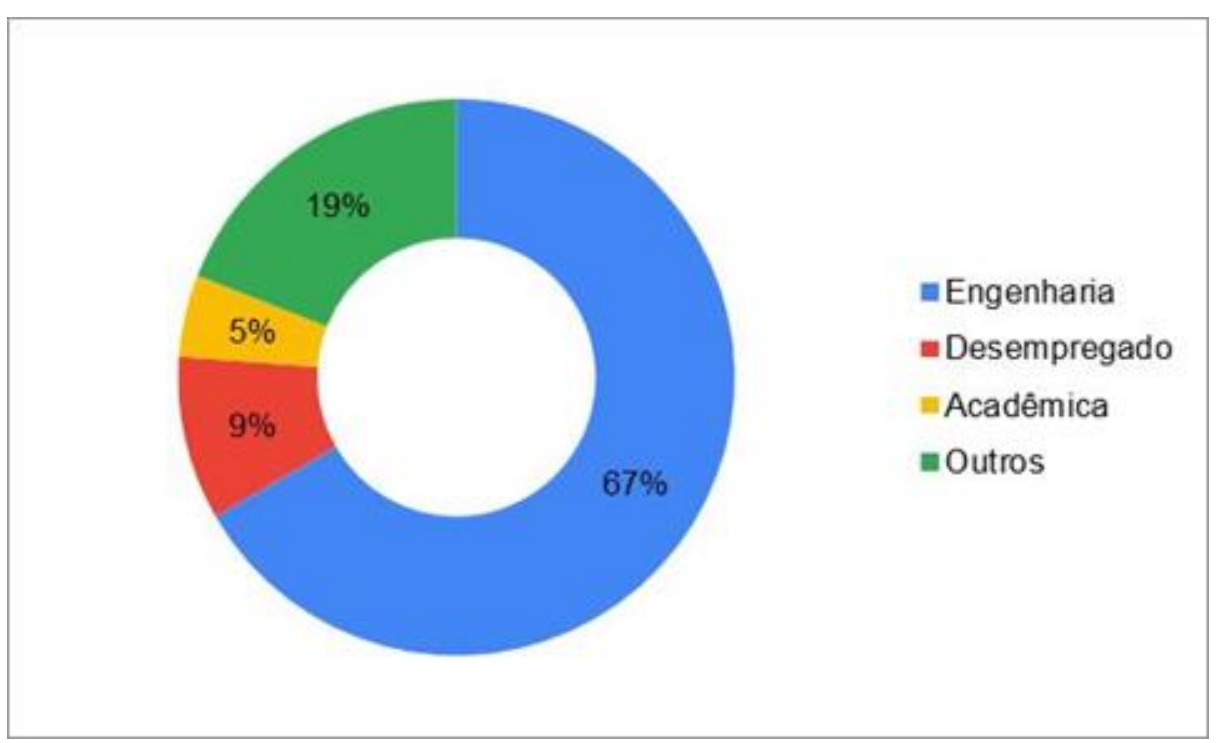

Figura 3 - Resultado da relação entre atuação e formação na visão dos egressos

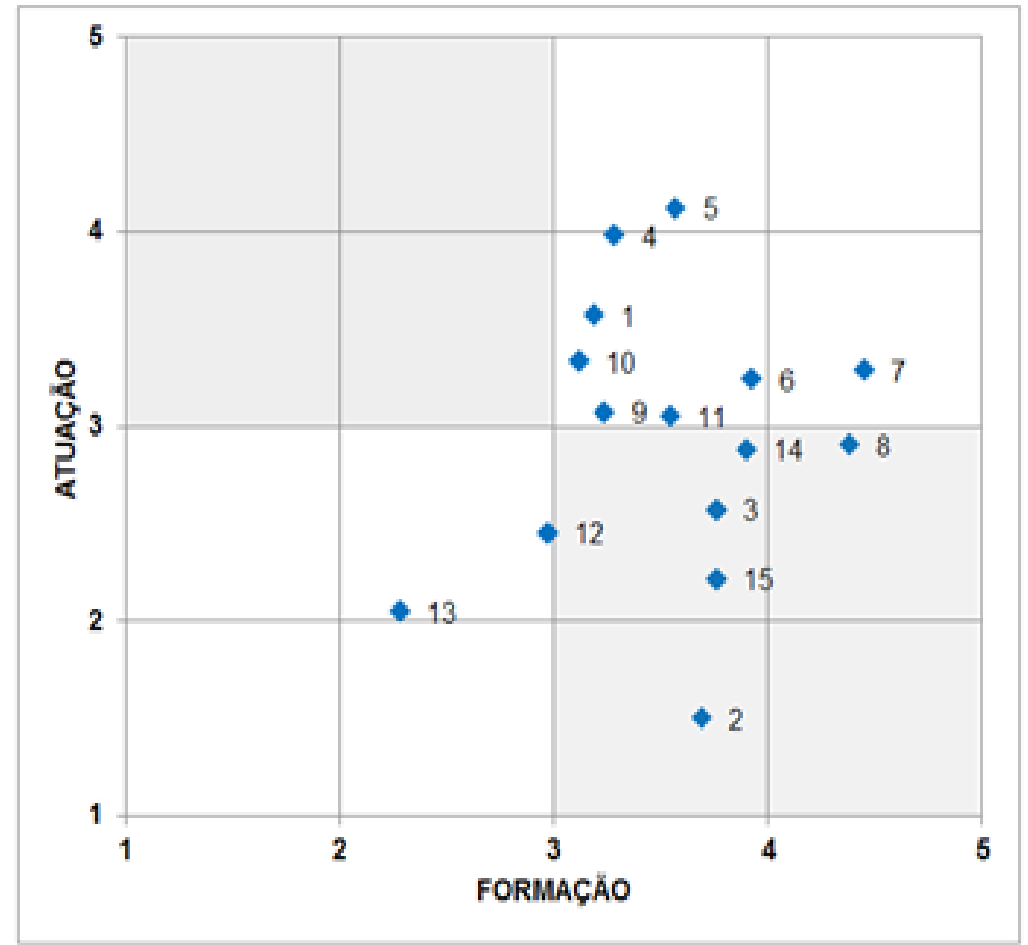

Administraçăo e Economia

2 Cálculo Numérico

3 Cartografia e Geoprocessamento

4 Construçăo $\mathrm{Civil}$

5 Desenho técnico e digital

6 Estruturas

7 Fundaçōes e Obras de Terra

8 Geologia e Mecánica dos solos

9 Hidraulica e Hidrologia

10 Legislaçăo e Segurança do Trabalho

11 Meio ambiente e Sustentabilidade

12 Planejamento Urbano

13 Programação

14 Saneamento e Residuos Sólidos

15 Planejamento e Infraestrutura de Transportes

Destaca-se que a grande maioria das áreas investigadas obteve média alta, demonstrando que a capacitação que receberam nelas foi satisfatória e que essas áreas têm grande utilidade no mercado profissional. Este resultado vai ao encontro das competências específicas propostas pelo NDE-ECI, visto que as áreas de maior 
pontuação estão relacionadas com os campos indicados nessas competências (Construção Civil, Estruturas, Geotecnia, Hidrotecnia e Transportes).

Verifica-se que apenas a área de "Programação" foi apontada como insatisfatória e de baixa utilidade. Como apontado anteriormente, a maioria dos respondentes não foi para a área acadêmica, provável motivo pelo qual este tópico tenha recebido nota baixa em termos de utilização na vida profissional. Entretanto, de acordo com as Diretrizes Curriculares Nacionais dos Cursos de Graduação em Engenharia, trata-se de conteúdo básico que deve ser contemplado em todas as habilitações. Assim, o NDE vislumbra identificar quais as metodologias mais adequadas para melhorar a formação de seus egressos.

O questionário também contemplou questões discursivas, nas quais os egressos se manifestaram sobre os assuntos que gostariam de ter se aprofundado mais ou aqueles que não se mostraram tão úteis no mercado de trabalho, como também puderam dar sugestões sobre a matriz curricular. As respostas indicaram que todas as áreas estudadas foram importantes para sua formação, tendo sido apontada uma maior fragilidade do curso em relação à área de construção civil, ao uso de ferramentas computacionais e a necessidade de uma maior aproximação da teoria com a prática. Nesse sentido, para atender esta demanda foram incorporadas as metodologias ativas de aprendizagem e disciplinas optativas inovadoras no novo projeto pedagógico.

$\mathrm{Na}$ Figura 4 mostra-se a nuvem de substantivos elaborada a partir das respostas discursivas dos egressos no questionário, na qual fica demonstrada a necessidade de formação nas cinco áreas apontadas pelo NDE no perfil do egresso.

Figura 4 - Nuvem de substantivos das respostas discursivas

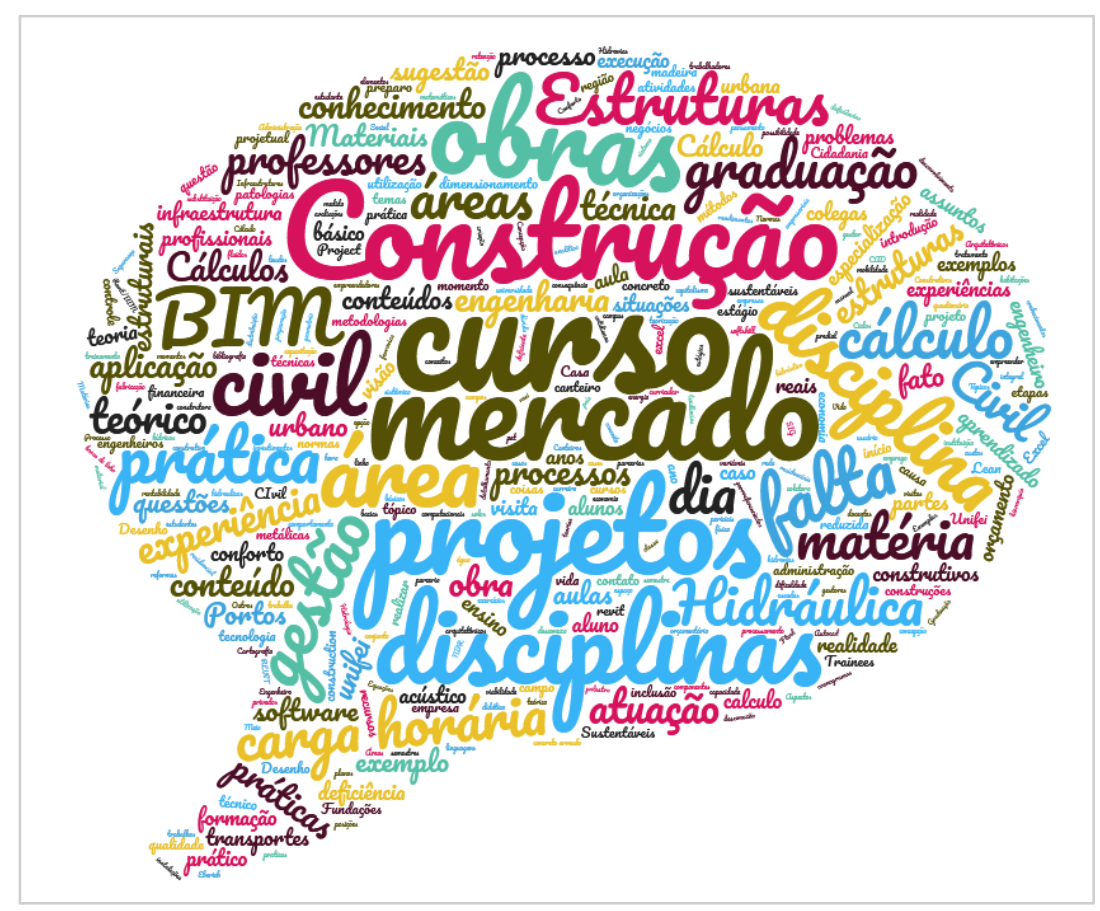

\section{CONSIDERAÇÕES FINAIS}

O perfil do egresso do curso de graduação em Engenharia Civil da Unifei foi definido com base nos campos de exercício profissional, na atuação dos egressos, nas demandas de mercado e nas discussões com os professores do curso.

Assim, o engenheiro civil graduado pela Unifei deve ser um profissional apto a atuar em todos os aspectos referentes às grandes áreas do conhecimento da Engenharia 
Civil, com qualificação para calcular, projetar, montar, construir, executar, fiscalizar e gerenciar serviços em diversos campos, entre estes: estruturas de concreto, elementos de fundações, estradas, planejamento de transportes, saneamento, hidráulica e meio ambiente, tendo sempre em vista aspectos atuais da engenharia e foco em tecnologias modernas e sustentáveis.

A partir da implantação do novo PPC, pretende-se aplicar novos questionários aos egressos, para avaliação permanente do curso. Como meta para a próxima avaliação será averiguada a necessidade de aglutinar e ou ampliar as áreas relativas às perguntas e tentar-se-á ampliar o número de respondentes ao questionário.

\section{Agradecimentos}

Agradecemos a Universidade Federal de Itajubá (Unifei) por todo apoio dado aos professores integrantes do Núcleo Docente Estruturante do curso de Engenharia Civil no desenvolvimento de suas atividades.

\section{REFERÊNCIAS}

MINISTÉRIO DA EDUCAÇÃO, INEP/DAES. Instrumento de avaliação de cursos de graduação - Presencial e a Distância - Reconhecimento e Renovação de Reconhecimento. 2017. [Online]. Disponível em: http://inep.gov.br/instrumentos

MINISTÉRIO DA EDUCAÇÃO, CNE/CES. Resolução N$^{\circ} 7$ de 18 de dezembro de 2018 Diretrizes para a Extensão na Educação Superior Brasileira. Brasília: Diário Oficial da União, Seção 1, pp. 49 e 50, 19 Dezembro 2018,. [Online]. Disponível em: http://portal.mec.gov.br/index.php?option=com docman\&view=download\&alias=104251rces007-18\&Itemid=30192.

MINISTÉRIO DA EDUCAÇÃO, CNE/CES. Resolução № 2 de 24 de abril de 2019 Diretrizes Curriculares Nacionais do Curso de Graduação em Engenharia. Brasília: Diário Oficial da União, Seção 1, pp. 43 e 44, 24 Abril 2019. [Online]. Disponível em: http://portal.mec.gov.br/index.php?option=com docman\&view=download\&alias=112681 rces002-19\&category slug=abril-2019-pdf\&ltemid=30192

MINISTÉRIO DA EDUCAÇÃO, CNE/CES. Resolução № 1, de 26 de março de 2021 Altera o Art. $9^{\circ}$, $\S 1^{\circ}$ da Resolução CNE/CES 2/2019 e o Art. 6 $6^{\circ}$ (1 da Resolução CNE/CES 2/2010, que institui as Diretrizes Curriculares Nacionais dos Cursos de Graduação de Engenharia, Arquitetura e Urbanismo. Brasília: Diário Oficial da União, Seção 1, p. 85, 29 Março 2021. [Online]. Disponível em: http://portal.mec.gov.br/index.php?option=com docman\&view=download\&alias=175301 rces001-21\&category slug=marco-2021-pdf\&ltemid=30192 


\title{
STUDY TO EVALUATE THE PROFILE OF UNIFEI'S CIVIL ENGINEERING COURSE
}

\begin{abstract}
The Civil Engineering course at the Federal University of Itajubá (Unifei) was proposed by the REUNI program and started in 2011. In 2019, motivated by discussions and changes in the National Curricular Guidelines of the undergraduate courses in Engineering, the Civil Engineering Structuring Teaching Center (NDE-ECI) started the process of restructuring the Pedagogical Course Project. The starting point was the discussions about the graduate's profile, culminating in the definition of the competences that are intended to develop for students during the course. The NDE-ECI proposed that graduates be qualified to work in the Civil Engineering five areas of knowledge, namely: Construction; Structures; Geotechnics, Hydrotechnics and Transportation. Thus, 14 competencies were established, the first eight being the general competences of the National Curricular Guidelines established in Art. 4 of the CNE/CES Resolution $\mathrm{N}^{\circ} 2$ of April 24,2019, and the other six defined by the NDE-ECl, one for each area of professional practice and another for the original project of the course, professional training with a focus on modern technologies and concerned with environmental issues. To validate the profile of the graduate proposed by the NDE, a questionnaire was applied to the graduates of the course. In the questionnaire, graduates in Civil Engineering gave their opinion, on a scale of 1 (worst) to 5 (best), on the major areas of basic and specific knowledge, evaluating the training they received throughout the course and its importance in their professional performance. The results showed that most areas are satisfactory in terms of training and that they are important for the job market. The research carried out corroborates the specific skills proposed by the NDE-ECl, since the areas with the highest scores are related to the fields indicated in them.
\end{abstract}

Keywords: egress, pedagogical project, construction, structures, geotechnics, hydrotechnics, transport, sustainability 